\title{
The $\delta 2$ Glutamate-Like Receptor Undergoes Similar Conformational Changes as Other Ionotropic Glutamate Receptors
}

\author{
David M. MacLean \\ Graduate Program in Pharmacology and Therapeutics, McGill University, Montreal, Quebec H3A 1Y6, Canada \\ Review of Hansen et al. (http://www.jneurosci.org/cgi/content/full/29/4/907)
}

Ligand-gated ion channels perform a remarkable feat: by coupling ligand binding to the opening of an electrical pore, they are able to unleash a flood of ions and decode chemical signals into appropriate electrical responses. Mapping out the precise conformational changes in this process for each type of receptor is challenging, but in the case of the ionotropic glutamate receptor (iGluR), our understanding has advanced considerably in the last decade. The iGluR family is composed of AMPA, kainate, NMDA, and delta subtypes, with a functional channel being composed of four subunits of a single type arranged as a dimer of dimers. How these individual subunits work together in a dimer is represented in Hansen et al. (2009) (their Fig. 7b). Essentially, each subunit possesses a clamshelllike agonist-binding domain (ABD) and is braced against its partner along the inner interface of the dimers. Agonist binding forces each subunit to "choose" a reaction: either the lower domain swings up in a move thought to pull open the channel's pore, or

Received March 26, 2009; revised April 17, 2009; accepted April 21, 2009. This work was supported by a Canadian Institute for Health Research Canada Graduate Scholarship. I thank Patricia Brown, Elizabeth Andrews, Mark Aurousseau, Pamela Lachance, and Drs. Mabel Chong and Derek Bowie for helpful discussions and Catie Lichten for a critical reading of this manuscript.

Correspondence should be addressed to David M. MacLean, Graduate Program in Pharmacology and Therapeutics, McIntyre Medical Sciences Building, McGill University, 3655 Promenade Sir William Osler, Montreal, QC H3A 1Y6, Canada. E-mail: David.maclean@mail.mcgill.ca.

DOI:10.1523/JNEUROSCI.1456-09.2009

Copyright $\odot 2009$ Society for Neuroscience $\quad$ 0270-6474/09/296767-02\$15.00/0 the upper jaw drops down, breaking the dimer interface in a motion believed to inactivate the channel despite the continued presence of the agonist. At present, it seems that members of the iGluR family roughly fit into this framework.

However, in many families there is a black sheep, and in the family of iGluRs, it is the enigmatic delta subunits Glu $\delta 1$ and $\delta 2$. Though classed as glutamate or glutamatelike receptors because of their relatively high amino acid sequence identity to other family members, the delta subunits stand apart from other glutamate receptors in two important respects. First and most striking, they do not bind glutamate. Second, the best described role of Glu $\delta 2$ (in long-term depression of parallel fiber inputs onto Purkinje cells) does not require the binding of any glutamate analogs (Yuzaki, 2005) or the ability to act as an ion channel (Kakegawa et al., 2007). However, if delta receptors are so different, can they fit in the same structurefunction framework established for the other family members?

Hansen et al. (2009) pursued this question by probing the structural changes a mutant form of Glu $\delta 2$ undergoes during increased activation via $\mathrm{Ca}^{2+}$ or inhibition via D-serine. Because Glu $\delta 2$ is electrically silent, the investigators used the lurcher point mutation, which endows Glu $\delta 2$ with constitutive ion channel activity. A previous study from this group (Naur et al., 2007) described the crystal structures of Glu $\delta 2$ and revealed that a pair of $\mathrm{Ca}^{2+}$ ions binds the ABD at the upper portion of the dimer interface [Hansen et al. (2009), their Fig. 4]. The stability of the homologous dimer interface in AMPA and kainate receptors is believed to be crucial in determining how readily these channels desensitize. Because the $\mathrm{Ca}^{2+}$ ions contact both partners of the dimer, the authors hypothesized that the binding of $\mathrm{Ca}^{2+}$ enhances the stability of a packed dimer conformation and ultimately results in a larger response. If true, this interpretation provides a structural correlate to the finding that $\mathrm{Ca}^{2+}$ potentiates Glu $\delta 2^{\mathrm{Lc}}$ currents (Wollmuth et al., 2000). Indeed, Hansen et al. (2009) replicated this finding and also observed that other divalents possess similar activity but only if they resemble $\mathrm{Ca}^{2+}$ in atomic size [Hansen et al. (2009), their Fig. 1]. Importantly, mutating the $\mathrm{Ca}^{2+}$ binding site to prevent divalent cation binding also prevented divalent potentiation, confirming that $\mathrm{Ca}^{2+}$ occupancy of this position is necessary for this effect [Hansen et al. (2009), their Fig. 4h].

Whereas $\mathrm{Ca}^{2+}$ (at $3 \mathrm{~mm}$ or less) (Wollmuth et al., 2000) potentiates Glu $\delta 2^{\mathrm{Lc}}$, D-serine inhibits Glu $\delta 2^{\mathrm{Lc}}$ currents. As described in a previous study (Naur et al., 2007), D-serine binds to the ABD of the Glu $\delta 2$ subunit, closing the domain upon itself. Interestingly, this form of domain closure is thought to activate all other types of iGluRs, but D-serine inhibits current flow through Glu $\delta 2^{\mathrm{Lc}}$ [Hansen et al. 
(2009), their Fig. 2]. The authors propose that $\mathrm{D}$-serine binding leads to a rearrangement of the dimer interface, reducing current through the channel in a mechanism akin to desensitization in iGluRs. Mutations to the D-serine-binding site found in crystal structures eliminated the inhibitory action [Hansen et al. (2009), their Fig. 2], indicating that it is attributable to D-serine binding to the ABD and not elsewhere. Moreover, introducing disulphide bonds to crosslink the subunits at the dimer interface showed that D-serine's inhibitory action requires rearrangement at this interface [Hansen et al. (2009), their Fig. 5].

These experiments have put forth a structural description of how $\mathrm{Ca}^{2+}$ can increase $\mathrm{Glu} \delta 2^{\mathrm{Lc}}$ activity by stabilizing its dimer interface, whereas D-serine has the opposite effect, decreasing activity by promoting dimer separation. So how do these two modulators compete with each other? In Hansen et al. (2009) (their Fig. 3 and their Fig. 4), they show that as one increases D-serine, and thus promotes dimer instability, one needs increasing amounts of $\mathrm{Ca}^{2+}$ to sustain a given level of potentiation. Similarly, the amount of D-serine needed to inhibit the channel increases with $\mathrm{Ca}^{2+}$ concentration. Addition of $\mathrm{Ca}^{2+}$ also changes the steepness of the D-serine dose-"response" curves. As seen in Hansen et al. (2009), their Figure $3 a$ and their Table 1, the Hill coefficient, $n$, which describes the steepness of the curve, shifts from having a value of near 1 in the absence of $\mathrm{Ca}^{2+}$ to 2 in the presence of $10 \mathrm{mM} \mathrm{Ca}^{2+}$. However, it is difficult to infer additional mechanistic information from this shift.

It is curious that in Glu $\delta 2$ crystal structures, D-serine, induces the same form of domain closure typically seen for agonists at other iGluRs yet acts as an inhibitor at Glu $\delta 2^{\text {Lc }}$. One explanation could be that mutant Glu $\delta 2^{\mathrm{Lc}}$ channel behaves differently than predicted by the Glu $\delta 2 \mathrm{ABD}$ crystal structure. The authors suggest another possibility: that structural constraints prevent D-serine from attaining its full potential as an agonist. They focus on a pair of Cys residues (C756 and C811) fond in both AMPA and NMDA receptors. Previous work has shown that breaking this disulfide bond with dithiothreitol in NMDAR promotes activation (Choi et al., 2001), and removing the bond via mutation in AMPARs increases agonist potency (Abele et al., 1998). Therefore, the authors suggest that as in other iGluRs, this bond may tonically attenuate the activation of Glu $\delta 2^{\mathrm{Lc}}$. As predicted, mutating one residue of the pair (C811S) to prevent the formation of the disulphide bond appeared to remove this tonic attenuation and converted D-serine into a weak partial agonist at Glu $\delta 2^{\mathrm{Lc}}$ receptors [Hansen et al. (2009), their Fig. 6, bottom left] and a strong agonist when the dimer interface was strengthened using the previously characterized engineered cross-links [Hansen et al. (2009), their Fig. 6, bottom right]. However, none of these manipulations endowed the wild-type Glu $\delta 2$ receptor with any detectable activity in either the presence or absence of $\mathrm{D}$-serine or $\mathrm{Ca}^{2+}$.

In light of the findings of Hansen et al. (2009), it appears that Glu $\delta 2$ is capable of conformational changes similar to those of its iGluR cousins, despite their differing functional roles. However, an important caveat in this and other studies of Glu $\delta 2$ is the use of the lurcher mutation, which may have unpredictable effects on the now-functional channel's behavior. In AMPA receptors, the lurcher mutation dramatically increases agonist efficacy (Taverna et al., 2000), and yet in the other Glu $\delta$ subunit, Glu $\delta 1$, the effects of this mutation are minor if present at all (Schmid and Hollmann, 2008). Because it is unclear precisely how the lurcher mutation works, the conformational changes in response to ligand binding to Glu $\delta 2^{\mathrm{Lc}}$ may be subtly or, perhaps, substantially different from those in wildtype.

Apart from this caveat, this study raises an interesting question about Glu $\delta 2$ 's mechanism of action. This subunit undergoes conformational changes like other iGluRs, but unlike them does not require a functioning pore for one of its major roles (Kakegawa et al., 2007). The authors highlight the possibility that Glu $\delta 2$ uses ligandinduced conformational changes to signal metabotropically. However, two findings appear to contradict this idea. First, many Glu $\delta 2$ functions do not require amino acidinduced conformational changes, since a mutant Glu $\delta 2$ which was altered to prevent amino acids such as D-serine from binding works just as well as a wild-type Glu $\delta 2$ (Yuzaki, 2005). Second, a recent study has revealed that Glu $\delta 2$ can induce presynaptic terminal differentiation even without its $\mathrm{ABD}$, the site of both $\mathrm{D}$-serine and $\mathrm{Ca}^{2+}$ binding (Kuroyanagi et al., 2009). On the surface, these findings argue against Glu $\delta 2$ using ligand-induced conformational change for metabotropic signaling. However, it is possible that GluR $\delta 2$ has additional unexamined roles which require D-serine, $\mathrm{Ca}^{2+}$, or some as yet unidentified ligand. It is also possible that ligand-induced conformational changes are not necessary for initiating signaling but rather modulate existing signals. If so, might other iGluRs share this common property of electrically independent signaling, such as the metabotropic action described for certain kainate receptors (Ruiz et al., 2005)? Investigating this most unusual of the iGluRs could ultimately provide insight into the capabilities of the whole family.

\section{References}

Abele R, Lampinen M, Keinänen K, Madden DR (1998) Disulfide bonding and cysteine accessibility in the alpha-amino-3-hydroxy-5methylisoxazole-4-propionic acid receptor subunit GluRD. Implications for redox modulation of glutamate receptors. J Biol Chem 273:25132-25138.

Choi Y, Chen HV, Lipton SA (2001) Three pairs of cysteine residues mediate both redox and $\mathrm{Zn}^{2+}$ modulation of the NMDA receptor. J Neurosci 21:392-400.

Hansen KB, Naur P, Kurtkaya NL, Kristensen AS, Gajhede M, Kastrup JS, Traynelis SF (2009) Modulation of the dimer interface at ionotropic glutamate-like receptor $\delta 2$ by $\mathrm{D}$-serine and extracellular calcium. J Neurosci 29:907-917.

Kakegawa W, Kohda K, Yuzaki M (2007) The delta2 'ionotropic' glutamate receptor functions as a non-ionotropic receptor to control cerebellar synaptic plasticity. J Physiol 584:89-96.

Kuroyanagi T, Yokoyama M, Hirano T (2009) Postsynaptic glutamate receptor $\delta$ familiy contributes to presynaptic terminal differentiation and establishment of synaptic transmission. Proc Natl Acad Sci USA 106:4912-4916.

Naur P, Hansen KB, Kristensen AS, Dravid SM, Pickering DS, Olsen L, Vestergaard B, Egebjerg J, Gajhede M, Traynelis SF, Kastrup JS (2007) Ionotropic glutamate-like receptor delta2 binds d-ser and glycine. Proc Natl Acad Sci U S A 104:14116-14121.

Ruiz A, Sachidhanandam S, Utvik JK, Coussen F, Mulle C (2005) Distinct subunits in heteromeric kainate receptors mediate ionotropic and metabotropic function at hippocampal mossy fiber synapses. J Neurosci 25:11710-11718.

Schmid SM, Hollmann M (2008) To gate or not to gate: are the delta subunits in the glutamate receptor family functional ion channels? Mol Neurobiol 37:126-141.

Taverna F, Xiong ZG, Brandes L, Roder JC, Salter MW, MacDonald JF (2000) The lurcher mutation of an alpha-amino-3-hydroxy-5methyl-isoxazolepropionic acid receptor subunit enhances the potency of glutamate and converts and antagonist to an agoinst. J Biol Chem 275:8475-8479.

Wollmuth LP, Kuner T, Jatzke C, Seeburg PH, Heintz N, Zuo J (2000) The lurcher mutation identifies $\delta 2$ as an AMPA/KA like channel that is potentiated by $\mathrm{Ca}^{2+}$. J Neurosci 20:5973-5980.

Yuzaki M (2005) Transgenic rescue for characterizing orphan receptors: a review of $\delta 2$ glutamate receptor. Transgenic Res 14:117-121. 